\title{
MODELO HÍBRIDO DE ANÁLISE FINANCEIRA APLICADO NO APRIMORAMENTO DA INTELIGÊNCIA DE NEGÓCIOS
}

\section{HYBRID FINANCIAL ANALYSIS MODEL APPLIED TO IMPROVE BUSINESS INTELLIGENCE}

\author{
MODELO HÍBRIDO DE ANÁLISIS FINANCIERO APLICADO PARA MEJORAR LA INTELIGENCIA \\ EMPRESARIAL
}

Denis Carlos Lima Costa1, Reno Silva Nooblath², Ana Beatriz das Neves da Silva ${ }^{3}$, Aryadne Nunes Sousa ${ }^{3}$, Christopher Ferreira de Barros ${ }^{3}$, Jossylyn Dayanny Casseb Campos ${ }^{3}$, Kevin Nascimento de Souza ${ }^{3}$

e311103

https://doi.org/10.47820/recima21.v3i1.1103

\section{RESUMO}

Esta pesquisa visa aprimorar a gestão empreendedora, em especial àquelas vinculadas aos trabalhadores informais e autônomos que encontram dificuldades quando se deparam com a utilização da tecnologia de aplicativos mobile. Propõem-se um Sistema Híbrido como forma de auxílio na administração dos negócios. Esse sistema associa Ciência e Tecnologia viabilizado pelos conhecimentos da Matemática Financeira implementados em um dos softwares mais úteis na gerência de empreendimentos. A partir da interdisciplinaridade entre as áreas da Matemática, Economia, Lógica de Programação e Algoritmos, pretende-se demonstrar uma série de vantagens da utilização de aplicativos que auxiliam na gestão e organização dos recursos financeiros, proporcionando uma melhora do desempenho e controle de atividades comerciais, bem como nas tomadas de decisões, resultando em uma estratégia denominada Inteligência de Negócios.

PALAVRAS-CHAVE: Inteligência de negócios. Matemática financeira. Sistemas computacionais

\begin{abstract}
This research aims to improve entrepreneurial management, especially those linked to informal and selfemployed workers who encounter difficulties when faced with the use of mobile application technology. A Hybrid System is proposed as a way of helping to manage the business. This system combines Science and Technology, made possible by the knowledge of Financial Mathematics implemented in one of the most useful software in project management. From the interdisciplinarity between the areas of Mathematics, Economics, Programming Logic and Algorithms, it is intended to demonstrate a series of advantages of using applications that help in the management and organization of financial resources, providing an improvement in the performance and control of commercial activities, as well as in decisionmaking, resulting in a strategy called Business Intelligence.
\end{abstract}

\section{KEYWORDS: Business intelligence. Financial math. Computer systems}

\section{RESUMEN}

Esta investigación tiene como objetivo mejorar la gestión empresarial, especialmente aquellos vinculados a trabajadores informales y autónomos que encuentran dificultades ante el uso de la tecnología de aplicaciones móviles. Se propone un Sistema Híbrido como una forma de ayudar a gestionar el negocio. Este sistema combina Ciencia y Tecnología, hecho posible gracias al conocimiento de Matemática Financiera implementado en uno de los software más útiles en la gestión de proyectos. A partir de la interdisciplinariedad entre las áreas de Matemática, Economía, Lógica de Programación y Algoritmos, se pretende demostrar una serie de ventajas del uso de aplicaciones que ayuden en la gestión y organización de los recursos financieros, proporcionando una mejora en el desempeño y

\footnotetext{
1 Doutor em Sistemas de Energia; Pesquisador do grupo Gradiente de Modelagem Matemática e Simulação Computacional - GM²SC; Professor do IFPA - Campus Ananindeua no Bacharelado em Ciência e Tecnologia; Área de concentração: Matemática Computacional aplicada no aprimoramento da Inteligência Artificial.

${ }^{2}$ Cientista da Computação; Professor do IFPA - Campus Ananindeua; Coordenador do curso de Informática; Área de concentração: Inteligência Computacional.

${ }^{3}$ Discentes do Curso de Informática do Instituto Federal de Educação, Ciência e Tecnologia do Pará - IFPA Campus Ananindeua.
}

RECIMA21 - Ciências Exatas e da Terra, Sociais, da Saúde, Humanas e Engenharia/Tecnologia 


\section{RECIMA21 - REVISTA CIENTÍFICA MULTIDISCIPLINAR ISSN 2675-6218}

MODELO HÍBRIDO DE ANÁLISE FINANCEIRA APLICADO NO APRIMORAMENTO DA INTELIGÊNCIA DE NEGÓCIOS Denis Carlos Lima Costa, Reno Silva Nooblath, Ana Beatriz das Neves da Silva, Aryadne Nunes Sousa, Christopher Ferreira de Barros, Jossylyn Dayanny Casseb Campos, Kevin Nascimento de Souza

control de las actividades comerciales, así como en la toma de decisiones, dando como resultado una estrategia denominada Inteligencia de negocios.

PALABRAS-CLAVE: Inteligencia de negócios. Matemática financeira. Sistemas informáticos

\section{INTRODUÇÃO}

Nos últimos 50 anos, a agilidade organizacional, a capacidade de lidar com soluções rápidas e implacáveis, exigiram um aumento da velocidade de análises de informações, intrínsecas em um ambiente competitivo repleto de oportunidades imprevisíveis. Essa velocidade só pode ser alcançada por profissionais altamente qualificados e congruentes em Matemática e suas representações computacionais.

Segundo o relatório "De Olho nas Metas 2017", 85\% dos estudantes chegam ao final do Ensino Médio sem aprender o mínimo desejado em matemática (El, 2015). Essa estatística vem se repetindo a cada ano, no sentido de a Matemática ser alusiva a uma muralha no desenvolvimento profissional dos indivíduos.

Segundo Moterle et al. (2018), o sucesso das micro e pequenas empresas (MPEs) segue por um grande desafio porque seus titulares não possuem conhecimentos técnicos sobre gestão financeira, tendo em vista a priorização da atividade principal de seu empreendedorismo. Essa realidade afasta cada vez mais o empreendedor da busca por conhecimento, o que pode ancorá-lo em uma situação de pouca lucratividade ou até prejuízo.

Para Macedo et al. (2015), uma das partes principais é a análise de gastos, pois ter um controle do seu fluxo de caixa se torna indispensável para qualquer empresa, sendo incluída também a vida pessoal. Neste ponto é necessário que o agente administrador já tenha a maturidade e devida competência de separar os recursos financeiros do empreendimento e da vida pessoal, ainda mais sabendo da estreita relação de dependência entre ambas.

Para Oliveira et al. (2018), o conhecimento de gastos abre a possibilidade de um melhor planejamento de gestão financeira, possibilitando assim o surgimento de novas habilidades para a tomada de decisões mais seguras. Silva Neto et al. (2018) complementa Oliveira dizendo que a tecnologia pode ser uma auxiliadora, tendo em vista que os aplicativos móveis se tornam mais presentes no cotidiano, no entanto, reafirma que seu uso deve ser feito de forma consciente.

Para Costa et al. (2018), a integração entre os conceitos e as operações da Economia, viabilizada pela linguagem computacional, otimiza a gestão financeira.

El-Adaileh e Foster (2019) apresentam uma pesquisa para ajudar os futuros pesquisadores na identificação de estudos, a partir da revisão de literatura relevantes. Em ordem para identificar a importância de cada um dos fatores, como a taxa de juros, os autores realizaram uma análise de frequência aos elementos envolvidos nas aplicações financeiras. 


\section{RECIMA21 - REVISTA CIENTÍFICA MULTIDISCIPLINAR ISSN 2675-6218}

MODELO HÍBRIDO DE ANÁLISE FINANCEIRA APLICADO NO APRIMORAMENTO DA INTELIGÊNCIA DE NEGÓCIOS Denis Carlos Lima Costa, Reno Silva Nooblath, Ana Beatriz das Neves da Silva, Aryadne Nunes Sousa, Christopher Ferreira de Barros, Jossylyn Dayanny Casseb Campos, Kevin Nascimento de Souza

Costa et al. (2019) apresentam métodos matemáticos desenvolvidos em linguagem Python de computação. A estratégia permite implementar as funções da Matemática Financeira em um formato universal e didático.

Vaio et al. (2020) destacam as principais contribuições em uma revisão bibliográfica abrangente da relação fundamental entre a Inteligência Artificial suas implicações acadêmicas e gerenciais na formatação das mudanças culturais exigidas pelas empresas, a fim de atingir metas sustentáveis.

Cheng et al. (2020) expõem um conjunto de técnicas, da chamada Business Intelligence (Inteligência de Negócios), e sua definição como um processo voluntário pelo qual uma empresa pode verificar e absorver informações de um ambiente turbulento para detectar uma oportunidade disponível, minimizando as ameaças associadas à incerteza. A partir desses fundamentos foi possível identificar várias ações-chave, como por exemplo, a coleta de dados, a análise de dados, o compartilhamento e a disseminação de informações como proposta de implementação de funções da IN.

Rasiwala e Kohli (2021) explicam que, uma das principais razões para realizar uma pesquisa qualitativa nesta área foi explorar o nível de conhecimento matemático e o seu efeito sobre as operações bancárias tradicionais na análise das decisões mais informadas e sobre como enfrentar os desafios do empreendedorismo.

Costa et al. (2021) propõem a implementação de elementos de Matemática-Computacional em linguagem MATLAB de computação. Os autores apresentam uma variedade de possibilidades para Modelagem Matemática com indicações às operações relacionadas à gestão e tomada de decisão.

O presente trabalho apresenta um sistema computacional que pode ser, facilmente, aplicado na gestão financeira na empreitada dos trabalhadores informais do cenário nacional em contraste com suas principais deficiências organizacionais e de gestão empreendedora, cuja base é lastreada pelo concreto conhecimento em Matemática básica. $O$ foco desta pesquisa são as micro e pequenas empresas, pois estas geralmente começam sob comando de trabalhadores autônomos, os quais apresentam déficits que comprometem a eficaz administração de seus empreendimentos.

\section{INTELIGÊNCIA DE NEGÓCIOS}

Para Dubey et al. (2019), a Inteligência de Negócios (Business Intelligence) refere-se à integração de dados e à capacidade analítica. A integração de dados visa combinar dados observáveis que residem em diferentes fontes para gerar informações descritivas, como quem, o quê, quando e quanto os dados unificados afetam. A capacidade analítica está intimamente relacionada à tomada de decisão da empresa e pode ajudar a transferir as informações úteis para o conhecimento explícito com o envolvimento do empreendedorismo, o que é propício para a tomada de decisão.

A Inteligência de Negócios (IN) é um conjunto de mecanismos, que define um processo voluntário, por meio do qual um empreendedor será capaz de minerar e absorver informações de um ambiente financeiro, tendo como meta detectar uma oportunidade disponível para maximizar o seu lucro, minimizando as ameaças associadas à incerteza (CHENG et al., 2020). 


\section{RECIMA21 - REVISTA CIENTÍFICA MULTIDISCIPLINAR ISSN 2675-6218}

MODELO HÍBRIDO DE ANÁLISE FINANCEIRA APLICADO NO APRIMORAMENTO DA INTELIGÊNCIA DE NEGÓCIOS Denis Carlos Lima Costa, Reno Silva Nooblath, Ana Beatriz das Neves da Silva, Aryadne Nunes Sousa, Christopher Ferreira de Barros, Jossylyn Dayanny Casseb Campos, Kevin Nascimento de Souza

Entende-se que integração de dados e a capacidade analítica são as duas características importantes da IN que podem ajudar uma empresa a adquirir dados de negócios, assimilar as informações valiosas e, finalmente, indicar novos conhecimentos à uma tomada de decisão em processos que apresentem teorias financeiras e econômicas.

\section{METODOLOGIA}

A metodologia executada nesse artigo é, prevalentemente, estruturalista, em conformidade com Pereira et al. (2018). Os objetivos foram instaurados com a averiguação de um evento factual. Em seguida, ampliou-se ao espaço da abstração, através da concepção de modelos matemáticos e computacionais. Apresenta-se o propósito científico e em seguida se evidencia o resultado da investigação, conectada às informações a priori da realidade, idealizada e correlacionada com as condições e restrições econômicas.

Dantas (2021), manifesta em seu trabalho, duas ferramentas importantes para a Gestão de Projetos: o PMBok (Project Management Body of Knowledge), que delibera o gerenciamento de projetos mais tradicional; a outra ferramenta é o SCRUM, definida como sendo uma estrutura na qual as pessoas podem resolver problemas adaptativos complexos, ao mesmo tempo que entregam produtos do mais alto valor possível de maneira produtiva e criativa.

As fases categorizadas da metodologia utilizada nesse trabalho estão evidenciadas com aplicação do software PLANILHAS, contido na maioria dos dispositivos que usam o Sistema Operacional Android. O PLANILHAS apresenta diversas vantagens: possui as funções dos modelos matemáticos usados na análise financeira, é gratuito e pode ser executado sem a conexão com a internet.

O PLANILHAS é um aplicativo baseado na web que permite aos usuários criar, atualizar e modificar PLANILHAS e compartilhar os dados online e em tempo real. O PLANILHAS faz parte do pacote de aplicativos gratuitos que também incluem o DOCUMENTOS e o APRESENTAÇÕES.

As operações financeiras realizadas pelo Sistema Computacional Android (PLANILHAS) estão fundamentadas nas Equações (1), (2), (3), (4), (5) e (6), representadas a seguir. 


\section{RECIMA21 - REVISTA CIENTÍFICA MULTIDISCIPLINAR ISSN 2675-6218}

MODELO HÍBRIDO DE ANÁLISE FINANCEIRA APLICADO NO APRIMORAMENTO DA INTELIGÊNCIA DE NEGÓCIO Denis Carlos Lima Costa, Reno Silva Nooblath, Ana Beatriz das Neves da Silva, Aryadne Nunes Sousa, Carlos Lima Costa, Reno Silva Nooblath, Ana Beatriz das Neves da Silva, Aryadne Nunes Sousa,
Christopher Ferreira de Barros, Jossylyn Dayanny Casseb Campos, Kevin Nascimento de Souza

$$
V F=V P(1+T A X A)^{N P E R}
$$

$$
V F=\frac{\operatorname{Pgto}\left[(1+T A X A)^{N P E R}-1\right]}{T A X A}
$$

$$
V P=\frac{V F}{(1+T A X A)^{N P E R}}
$$

$$
V P=\frac{P g t o\left[(1+T A X A)^{N P E R}-1\right]}{T A X A(1+T A X A)^{N P E R}}
$$

$$
\begin{gathered}
\text { Pgto }=V P \frac{T A X A(1+T A X A)^{N P E R}}{(1+T A X A)^{N P E R}-1} \\
\text { Pgto }=V F \frac{T A X A}{(1+T A X A)^{N P E R}-1}
\end{gathered}
$$

Sendo,

$V F \rightarrow$ o valor futuro;

$V P \rightarrow$ o valor presente;

$T A X A \rightarrow$ a taxa de juros;

$N P E R \rightarrow$ o número de períodos;

$P g t o \rightarrow$ o valor do pagamento.

Nascimento (2021) propõe uma modelagem matemática como estratégia pedagógica em ambientes informatizados. Em seu trabalho, a autora obteve excelentes resultados, na perspectiva do ensino da Matemática, com o aporte tecnológico, pois, segundo ela, a interação do conteúdo com a realidade materializaram uma aprendizagem significativa.

Fundamentados nessa experiência, realizou-se uma Modelagem Matemática-Computacional que associa as equações das Ciências Econômicas, utilizadas pela Inteligência de Negócios, a um dos mais simples e robusto sistemas computacionais: o PLANILHAS. Devido ao seu fácil manuseio, disponibilidade e gratuidade, o Planilha se mostrou didático e eficiente, o que corrobora com a ideia de otimização do processo de ensino-aprendizagem multidisciplinar.

As Equações (1) e (2) poderão ser implementadas computacionalmente conforme o fluxograma apresentado na Figura 1. 


\section{RECIMA21 - REVISTA CIENTÍFICA MULTIDISCIPLINAR ISSN 2675-6218}

MODELO HÍBRIDO DE ANÁLISE FINANCEIRA APLICADO NO APRIMORAMENTO DA INTELIGÊNCIA DE NEGÓCIOS Denis Carlos Lima Costa, Reno Silva Nooblath, Ana Beatriz das Neves da Silva, Aryadne Nunes Sousa, Christopher Ferreira de Barros, Jossylyn Dayanny Casseb Campos, Kevin Nascimento de Souza

Figura 1: Fluxograma para execução do cálculo do VF

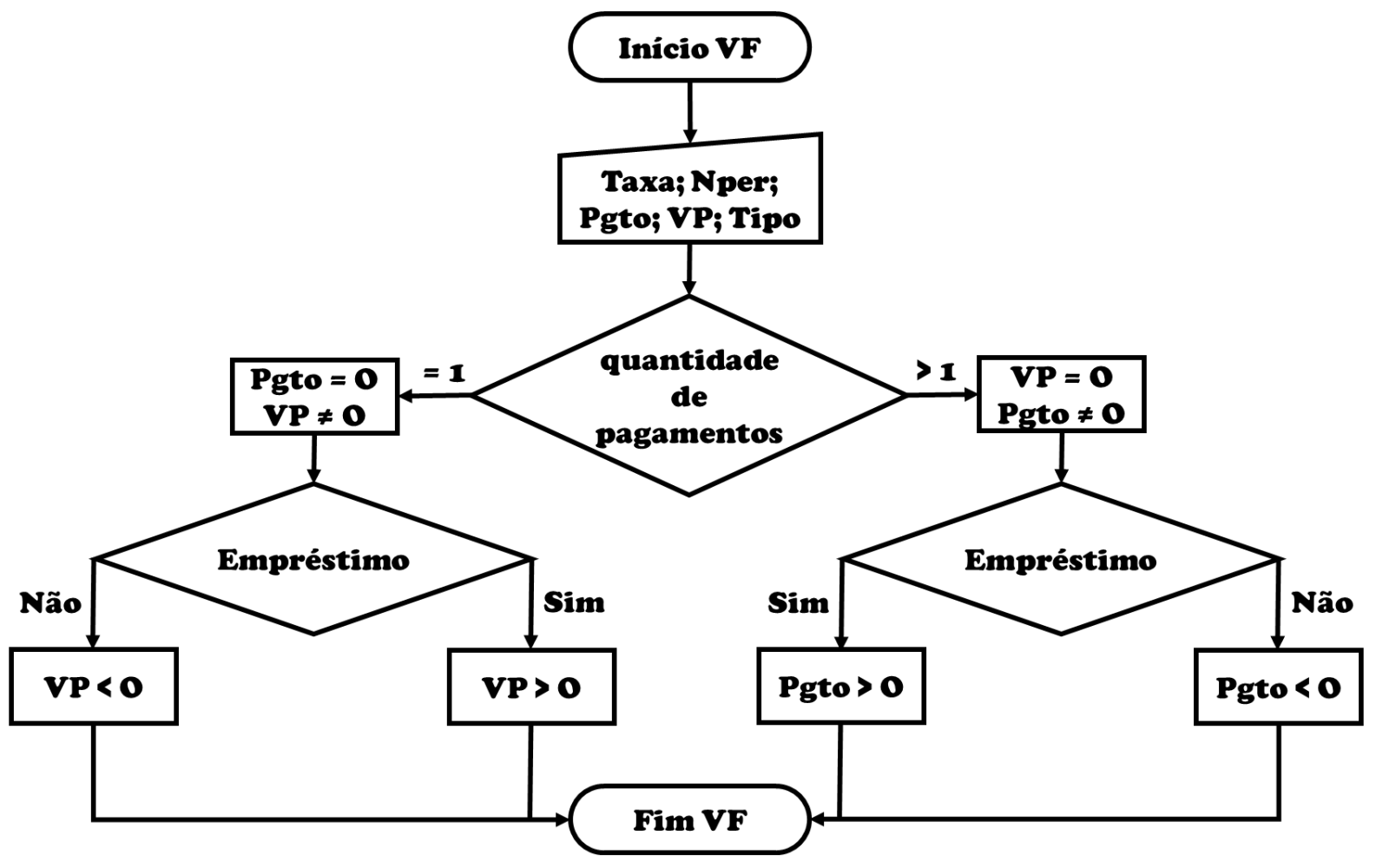

Fonte: Autores (2022).

As Equações (3) e (4) poderão ser implementadas computacionalmente conforme o fluxograma apresentado na Figura 2. 


\section{RECIMA21 - REVISTA CIENTÍFICA MULTIDISCIPLINAR ISSN 2675-6218}

MODELO HÍBRIDO DE ANÁLISE FINANCEIRA APLICADO NO APRIMORAMENTO DA INTELIGÊNCIA DE NEGÓCIOS Denis Carlos Lima Costa, Reno Silva Nooblath, Ana Beatriz das Neves da Silva, Aryadne Nunes Sousa, Christopher Ferreira de Barros, Jossylyn Dayanny Casseb Campos, Kevin Nascimento de Souza

Figura 2: Fluxograma para execução do cálculo do VP

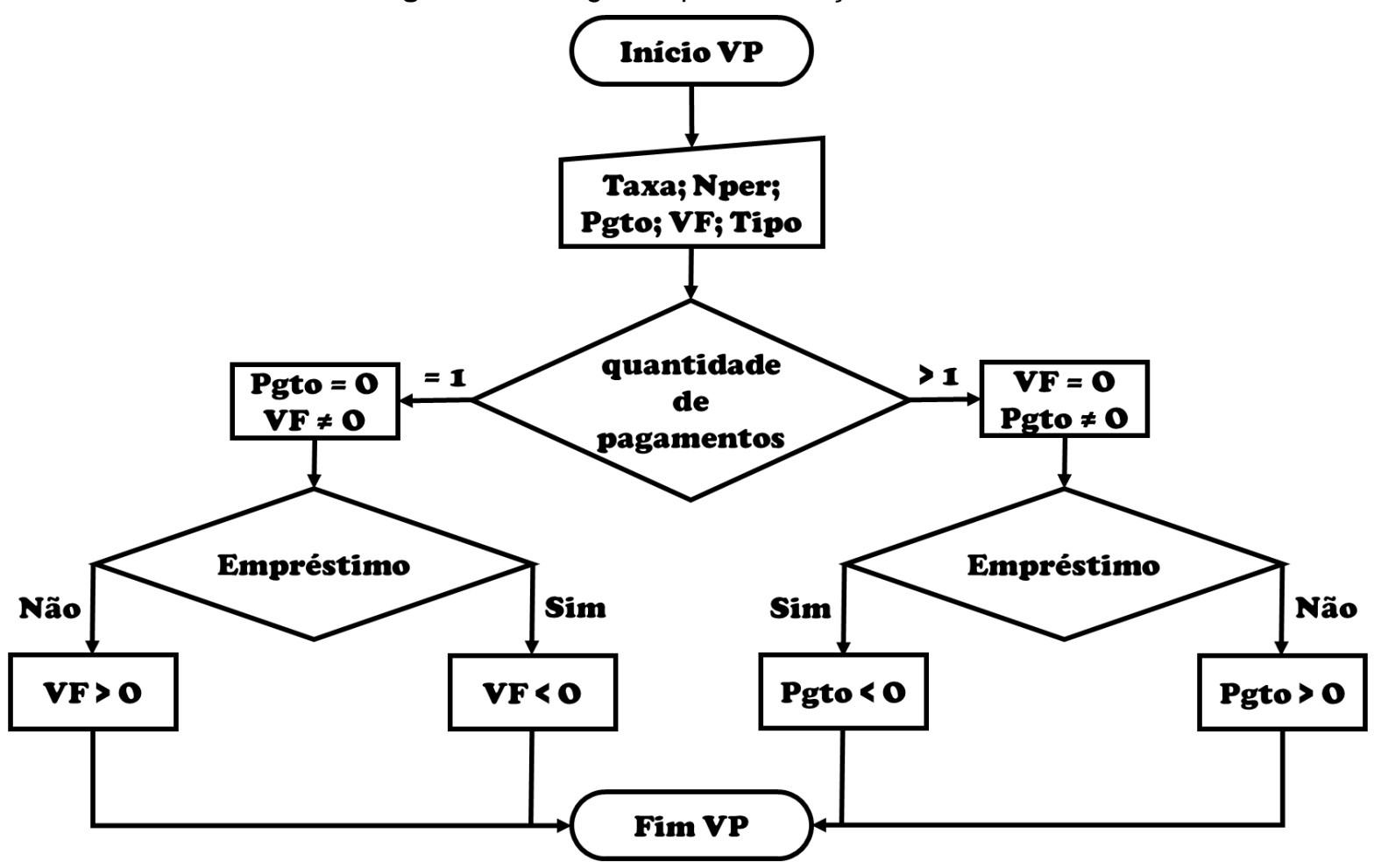

Fonte: Autores (2022).

As Equações (5) e (6) poderão ser implementadas computacionalmente conforme o fluxograma apresentado na Figura 3. 
RECIMA21 - REVISTA CIENTÍFICA MULTIDISCIPLINAR ISSN 2675-6218

MODELO HÍBRIDO DE ANÁLISE FINANCEIRA APLICADO NO APRIMORAMENTO DA INTELIGÊNCIA DE NEGÓCIOS Denis Carlos Lima Costa, Reno Silva Nooblath, Ana Beatriz das Neves da Silva, Aryadne Nunes Sousa, Christopher Ferreira de Barros, Jossylyn Dayanny Casseb Campos, Kevin Nascimento de Souza

Figura 3: Fluxograma para execução do cálculo do PGTO

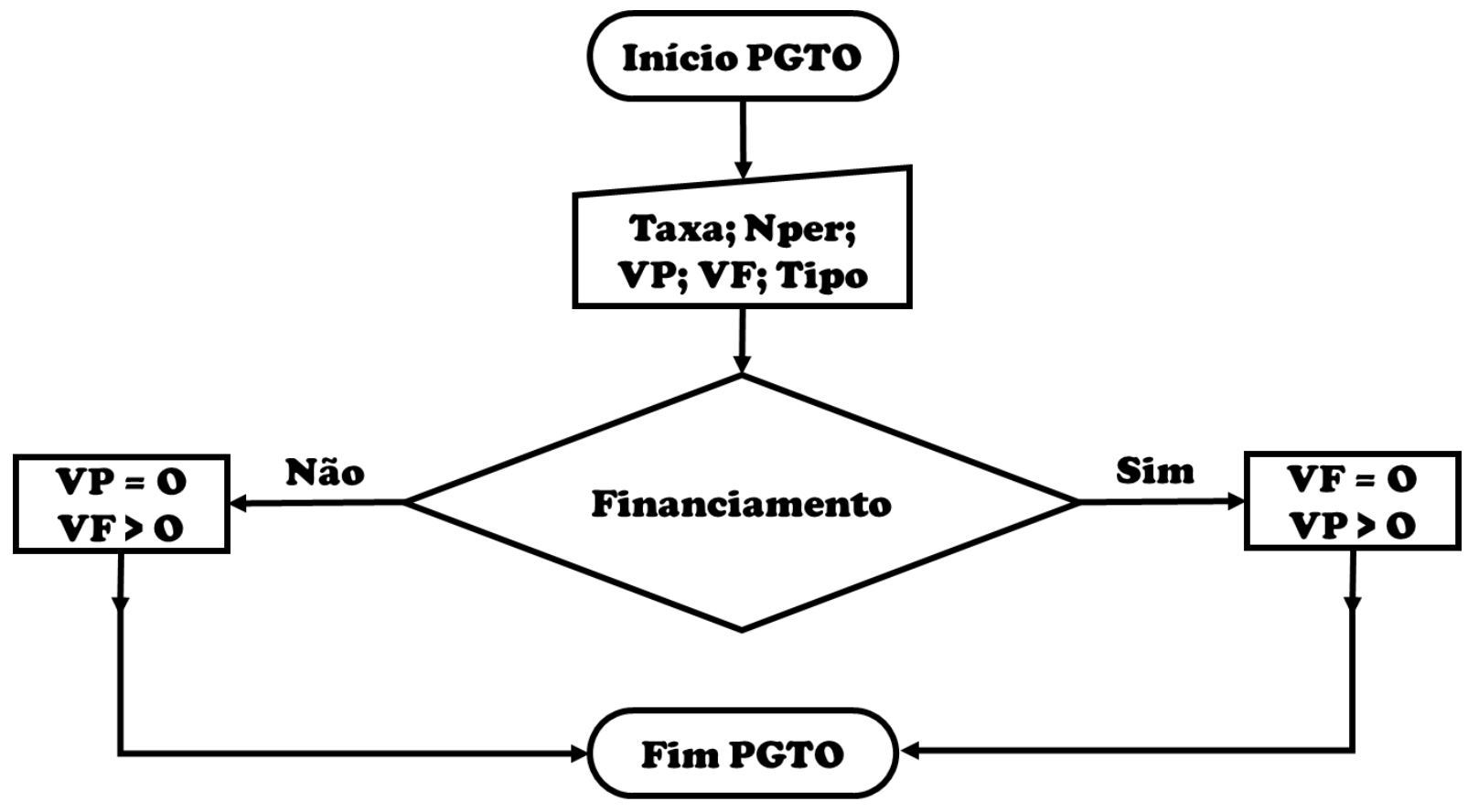

Fonte: Autores (2022).

\section{RESULTADOS E DISCUSSÕES}

Neste artigo foram utilizados 3 (três) estudos de caso: Caso i, refere-se ao cálculo do Valor Futuro (VF); Caso ii, refere-se ao cálculo do Valor Presente (VP); Caso iii, refere-se ao cálculo do Valor do Pagamento (PGTO).

A resolução Matemática-Computacional, de todos os Casos, foi realizada mediante a aplicação do PLANILHAS, correlacionado as operações com as equações da Matemática Financeira, estimulando, dessa forma, a Inteligência de Negócios.

A Figura 4 representa, respectivamente, o ícone e a interface desse software.

Figura 4: Ícone e interface do PLANILHAS

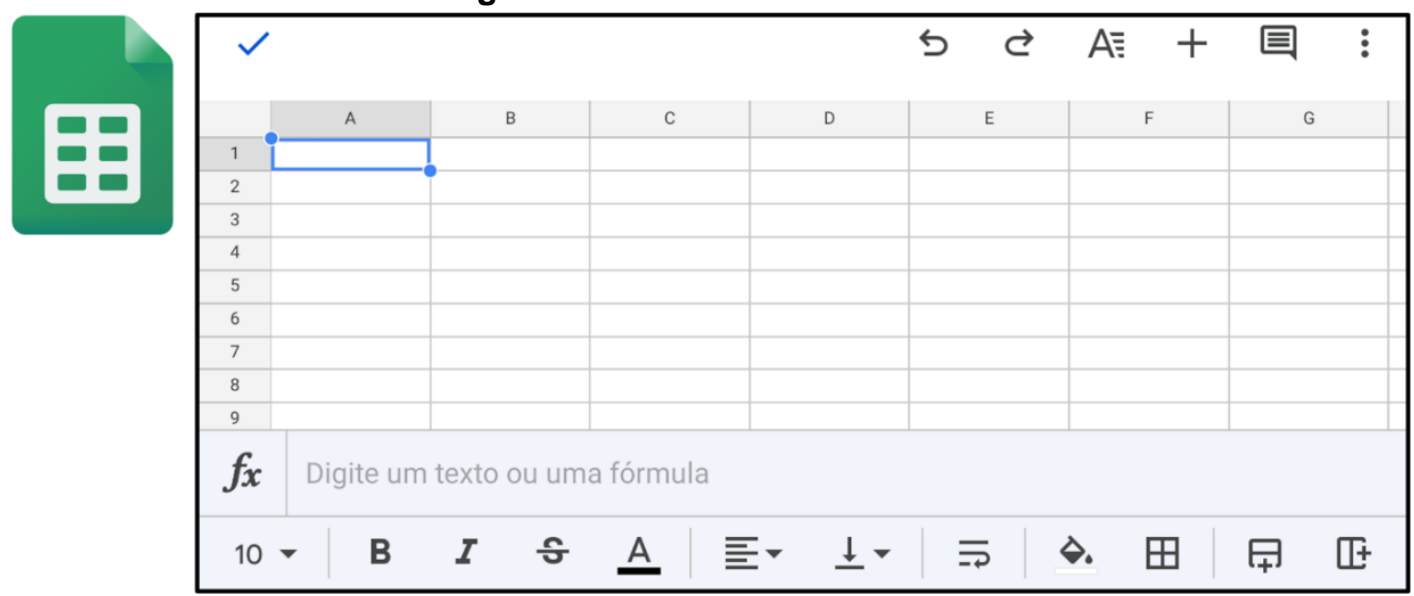

Fonte: Autores (2022).

RECIMA21 - Ciências Exatas e da Terra, Sociais, da Saúde, Humanas e Engenharia/Tecnologia 


\section{RECIMA21 - REVISTA CIENTÍFICA MULTIDISCIPLINAR ISSN 2675-6218}

MODELO HÍBRIDO DE ANÁLISE FINANCEIRA APLICADO NO APRIMORAMENTO DA INTELIGÊNCIA DE NEGÓCIOS Denis Carlos Lima Costa, Reno Silva Nooblath, Ana Beatriz das Neves da Silva, Aryadne Nunes Sousa, Christopher Ferreira de Barros, Jossylyn Dayanny Casseb Campos, Kevin Nascimento de Souza

Caso $i$ : A taxa de juros de $3 \%$ ao mês corrige uma operação financeira durante 24 meses. Estimar o Valor Futuro (VF), nas seguintes condições:

a. O capital de capital de $\mathrm{R} \$ 3000,00$ foi aplicado em uma instituição financeira.

Como há apenas um pagamento, tem-se, Pgto $=0$. Não sendo um empréstimo VP $=-3000$.

A Figuras 5 apresenta a composição das variáveis financeiras no PLANILHAS: $\boldsymbol{f} \boldsymbol{x}$. Lembrando que essa relação entre CÉLULA e a grandeza financeira, é opcional.

Figura 5: Valores inseridos no PLANILHAS

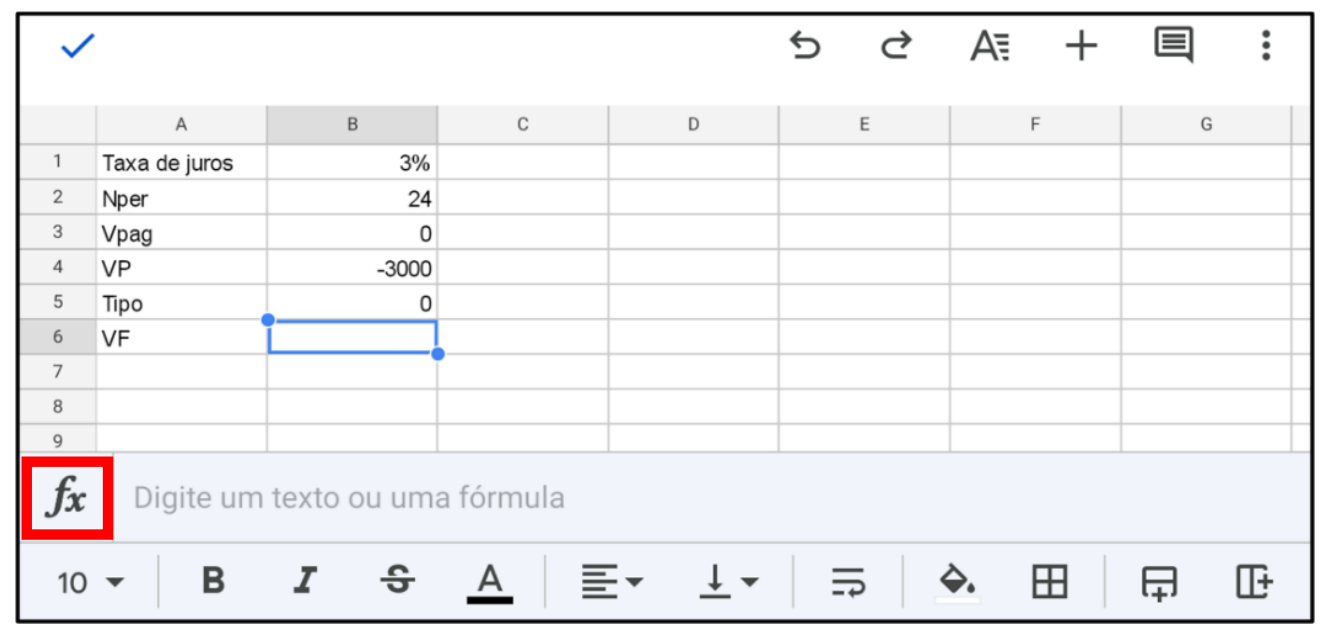

Fonte: Autores (2022).

Contudo, ressalta-se que essa representação favorece o processo de ensino-aprendizagem, pois cria-se uma conexão com outras áreas do conhecimento como Matrizes, Funções Polinomiais, Funções Não-Polinomiais, Estrutura de Dados, Lógica de Programação e Algoritmos, proporcionando, desta forma, uma interdisciplinaridade.

A variável TIPO corresponde ao momento do pagamento: POSTECIPADO $($ TIPO $=0)$ ou ANTECIPADO $(T I P O=1)$. No TIPO $=0$, o pagamento será efetuado no final do período. Enquanto que, no $T I P O=1$, o pagamento será efetuado no início do período. Os estudos realizados nessa pesquisa consideram todos os modelos POSTECIPADOS.

A Figura 6 mostra a estrutura para acessar as funções financeiras: FINANÇAS no PLANILHAS. 


\section{RECIMA21 - REVISTA CIENTÍFICA MULTIDISCIPLINAR ISSN 2675-6218}

MODELO HÍBRIDO DE ANÁLISE FINANCEIRA APLICADO NO APRIMORAMENTO DA INTELIGÊNCIA DE NEGÓCIOS Denis Carlos Lima Costa, Reno Silva Nooblath, Ana Beatriz das Neves da Silva, Aryadne Nunes Sousa, Christopher Ferreira de Barros, Jossylyn Dayanny Casseb Campos, Kevin Nascimento de Souza

Figura 6: Funções financeiras no PLANILHAS

\begin{tabular}{|l|}
\hline$\times \quad$ Inserir função \\
Estatística \\
Filtro \\
Finanças \\
Google \\
Informações
\end{tabular}

Fonte: Autores (2022).

A Figura 7 mostra a sequência para acessar a função financeira VF referente ao VALOR FUTURO.

Figura 7: Funções financeiras no PLANILHAS

\begin{tabular}{|l|}
\hline$\leftarrow$ Finanças \\
Calcula a taxa de juros efetiva gerada quando um investimento é comprado por um preço e ve... \\
TIR \\
Calcula a taxa interna de retorno sobre um investimento com base em uma série de fluxos de... \\
VF \\
Calcula o valor futuro de um investimento anual, com base em pagamentos periódicos e const... \\
VFPLANO \\
Calcula o valor futuro de capital com base em uma série especificada de taxas de juros possiv... \\
\hline
\end{tabular}

Fonte: Autores (2022).

A função $V F$, no PLANILHAS, receberá como input os valores, no formato numérico ou com as respectivas CÉLULAS: $=\boldsymbol{V F}(\boldsymbol{T a x a} ; \boldsymbol{N p e r} ; \boldsymbol{P g t o} ; \boldsymbol{V P}, \boldsymbol{T i p o})$.

A Figura 8a mostra $\boldsymbol{V} \boldsymbol{F}$ com os endereços das CÉLULAS e a Figura 8b, mostra o valor calculado de $\boldsymbol{V F}$. 


\section{RECIMA21 - REVISTA CIENTÍFICA MULTIDISCIPLINAR ISSN 2675-6218}

MODELO HÍBRIDO DE ANÁLISE FINANCEIRA APLICADO NO APRIMORAMENTO DA INTELIGÊNCIA DE NEGÓCIOS Denis Carlos Lima Costa, Reno Silva Nooblath, Ana Beatriz das Neves da Silva, Aryadne Nunes Sousa, Christopher Ferreira de Barros, Jossylyn Dayanny Casseb Campos, Kevin Nascimento de Souza

Figura 8a: Input para VF

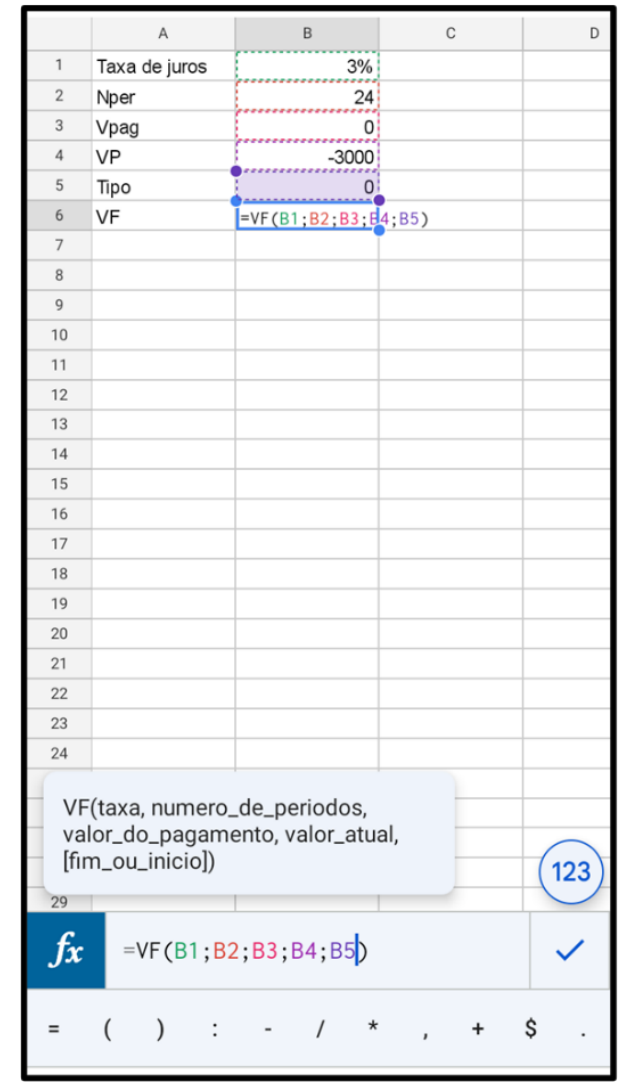

Figura 8b: Input pata VF

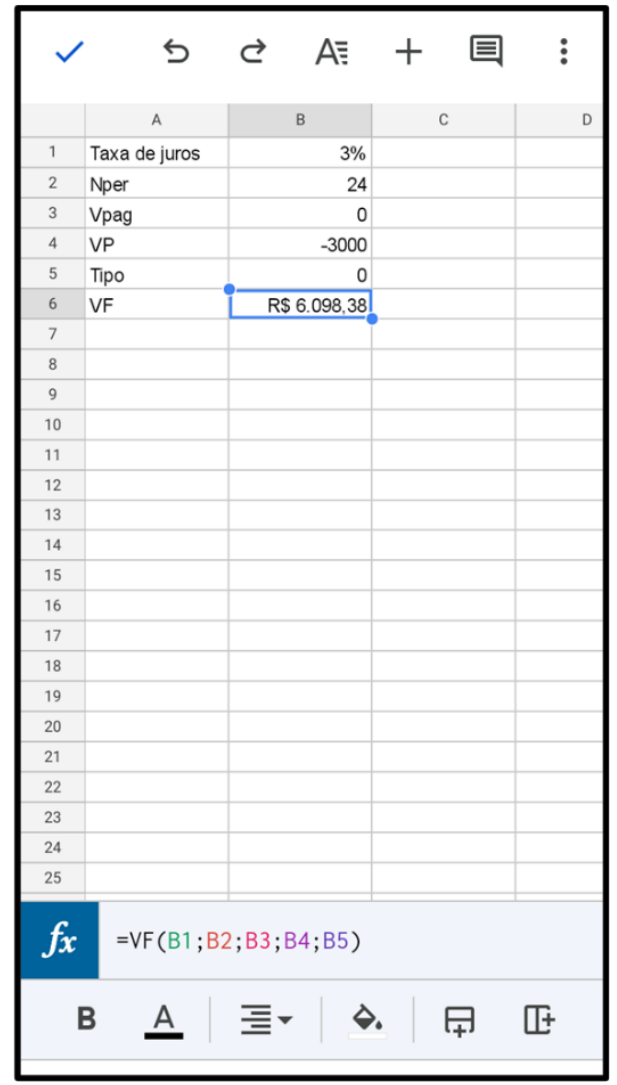

Fonte: Autores (2022).

A resolução algébrica poderá ser obtida mediante a aplicação da Equação (1).

No Caso i-a, a cliente deverá receber o valor de $\mathrm{R} \$ 6.098,38$ à instituição financeira, logo, $\boldsymbol{V F}$ será positivo.

$$
\begin{gathered}
=V F(3 \% ; 24 ; 0 ;-3000 ; 0) \text { ou } \\
V F=-3000(1+0,03)^{24} \rightarrow 6.098,38
\end{gathered}
$$

b. O capital de $\mathrm{R} \$ 3000,00$ foi emprestado de uma instituição financeira.

Como há apenas um pagamento, tem-se, Pgto $=0$. Sendo um empréstimo $V P=3000$.

A resolução do Caso i-b irá diferir do Caso i-a, em relação ao sinal de VPe, consequentemente, no sinal de VF. No Caso $i-b$, a cliente deverá pagar o valor de $\mathrm{R} \$ 6.098,38$ à instituição financeira, logo, $V \boldsymbol{F}$ será negativo.

$$
\begin{gathered}
=V F(3 \% ; 24 ; 0 ; 3000 ; 0) \text { ou } \\
V F=3000(1+0,03)^{24} \rightarrow-6.098,38
\end{gathered}
$$

c. Foram recebidos, de uma instituição financeira, 24 parcelas de $R \$ 250,00$. A resolução algébrica poderá ser obtida mediante a aplicação da Equação (2). 


\section{RECIMA21 - REVISTA CIENTÍFICA MULTIDISCIPLINAR ISSN 2675-6218}

MODELO HÍBRIDO DE ANÁLISE FINANCEIRA APLICADO NO APRIMORAMENTO DA INTELIGÊNCIA DE NEGÓCIOS Denis Carlos Lima Costa, Reno Silva Nooblath, Ana Beatriz das Neves da Silva, Aryadne Nunes Sousa, Christopher Ferreira de Barros, Jossylyn Dayanny Casseb Campos, Kevin Nascimento de Souza

$$
=V F(3 \% ; 24 ; 250 ; 0 ; 0) \text { ou }
$$

$$
V F=\frac{250\left[(1+0,03)^{24}-1\right]}{0,03} \rightarrow-8.606,62
$$

Percebe-se que, como há 24 recebimentos, $P$ GTO é positivo; logo, VF será negativo, indicando que haverá um pagamento de $\mathrm{R} \$ 8.606,62$ à instituição financeira.

d. Foram pagos, a uma instituição financeira, 24 parcelas de $R \$ 250,00$.

$$
\begin{gathered}
=V F(3 \% ; 24 ;-250 ; 0 ; 0) \text { ou } \\
V F=\frac{-250\left[(1+0,03)^{24}-1\right]}{0,03} \rightarrow 8.606,62
\end{gathered}
$$

Percebe-se que, como há 24 pagamentos, PGTO é negativo; logo, VF será positivo, indicando que haverá um recebimento de $R \$ 8.606,62$ da instituição financeira.

Caso ii: A taxa de juros de $1,72 \%$ ao mês foi utilizada em uma movimentação financeira durante 3 anos. Estimar o Valor Presente (VP), nas seguintes condições:

a. O capital de $R \$ 12.000,00$ será o pagamento referente ao empréstimo.

Para todas as resoluções, o script diferirá no último comando: na escolha da função financeira. A Figura 9 expõe essa rotina.

Figura 9: Rotina para execução da função financeira
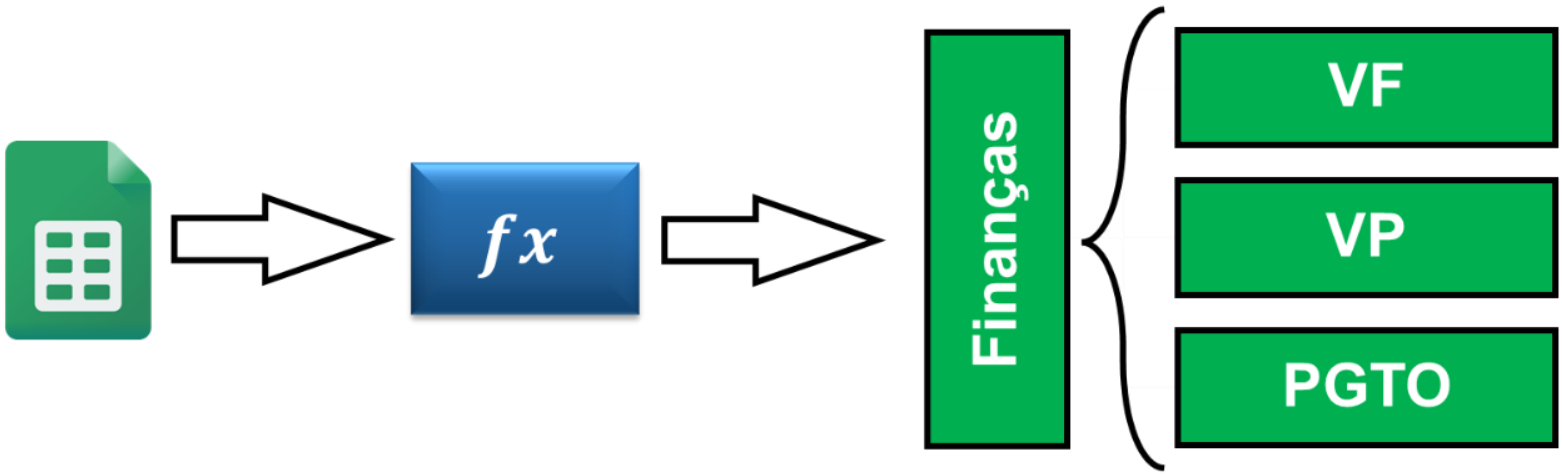

Fonte: Autores (2022).

A função $\boldsymbol{V P}$, no PLANILHAS, receberá como input os valores, no formato numérico ou com as

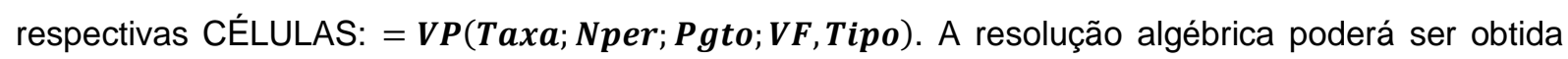
mediante a aplicação da Equação (3).

$$
\begin{gathered}
=V P(1,72 \% ; 36 ; 0 ;-12000 ; 0) \text { ou } \\
\quad V P=\frac{-12000}{(1+0,0172)^{36}} \rightarrow 6.494,60
\end{gathered}
$$




\section{RECIMA21 - REVISTA CIENTÍFICA MULTIDISCIPLINAR ISSN 2675-6218}

MODELO HÍBRIDO DE ANÁLISE FINANCEIRA APLICADO NO APRIMORAMENTO DA INTELIGÊNCIA DE NEGÓCIOS Denis Carlos Lima Costa, Reno Silva Nooblath, Ana Beatriz das Neves da Silva, Aryadne Nunes Sousa, Christopher Ferreira de Barros, Jossylyn Dayanny Casseb Campos, Kevin Nascimento de Souza

VF negativo ratifica pagamento; $\boldsymbol{V P}$ positivo confirma o recebimento.

b. Aplicar VP a fim de resgatar o capital de $R \$ 12.000,00$.

$$
\begin{aligned}
& =V P(1,72 \% ; 36 ; 0 ; 12000 ; 0) \text { ou } \\
& V P=\frac{12000}{(1+0,0172)^{36}} \rightarrow-6.494,60
\end{aligned}
$$

$\boldsymbol{V F}$ positivo confirma o recebimento; $\boldsymbol{V P}$ negativo ratifica pagamento.

c. Foram realizados 36 pagamentos de $R \$ 400,00$ cada, à intuição financeira. A resolução algébrica poderá ser obtida mediante a aplicação da Equação (4).

$$
\begin{gathered}
=V P(1,72 \% ; 36 ;-400 ; 0 ; 0) \text { ou } \\
V P=\frac{-400\left[(1+0,0172)^{36}-1\right]}{0,0172(1+0,0172)^{36}} \rightarrow 10.669,37
\end{gathered}
$$

PGTO negativo ratifica pagamentos; VP positivo confirma o recebimento.

d. Foram recebidas 36 parcelas de $R \$ 400,00$ cada, da intuição financeira.

$$
\begin{gathered}
=V P(1,72 \% ; 36 ; 400 ; 0 ; 0) \text { ou } \\
V P=\frac{400\left[(1+0,0172)^{36}-1\right]}{0,0172(1+0,0172)^{36}} \rightarrow-10.669,37
\end{gathered}
$$

PGTO positivo ratifica recebimentos; VP positivo confirma o pagamento.

Caso iii: A taxa de juros de 2,25\% ao mês foi utilizada em uma transação financeira durante 4,5 anos. Estimar o Valor do Pagamento efetuado mensalmente, nas seguintes condições:

a. Os pagamentos se referem às parcelas de um empréstimo de $R \$ 15.000,00$.

Para os Casos iii-a e iii-b, a função PGTO, no PLANILHAS, receberá como input os valores, no formato numérico ou com as respectivas CÉLULAS: = PGTO $(\boldsymbol{T a x a} ; \boldsymbol{N p e r} ; \boldsymbol{V P} ; \boldsymbol{V F}, \mathbf{T i p o})$. A resolução algébrica poderá ser obtida mediante a aplicação da Equação (5).

$$
\begin{gathered}
=P G T O(2,25 \% ; 54 ; 15000 ; 0 ; 0) \text { ou } \\
\text { PGTO }=15000 \frac{0,0225(1+0,0225)^{54}}{(1+0,0225)^{54}-1} \rightarrow-482,65
\end{gathered}
$$




\section{RECIMA21 - REVISTA CIENTÍFICA MULTIDISCIPLINAR ISSN 2675-6218}

MODELO HÍBRIDO DE ANÁLISE FINANCEIRA APLICADO NO APRIMORAMENTO DA INTELIGÊNCIA DE NEGÓCIOS Denis Carlos Lima Costa, Reno Silva Nooblath, Ana Beatriz das Neves da Silva, Aryadne Nunes Sousa, Christopher Ferreira de Barros, Jossylyn Dayanny Casseb Campos, Kevin Nascimento de Souza

$\boldsymbol{V P}$ positivo ratifica que foi um empréstimo; $\boldsymbol{P G T O}$ negativo indica que haverá pagamentos das parcelas desse empréstimo.

b. Os pagamentos se referem às parcelas de uma aplicação, cujo o montante será, de $\mathrm{R} \$$ 15.000,00. A resolução algébrica poderá ser obtida mediante a aplicação da Equação (6).

$$
\begin{gathered}
=P G T O(2,25 \% ; 54 ; 0 ; 15000 ; 0) \text { ou } \\
\text { PGTO }=15000 \frac{0,0225}{(1+0,0225)^{54}-1} \rightarrow-145,15
\end{gathered}
$$

VF positivo ratifica que haverá o recebimento de um capital; $P$ GTO negativo indica que os pagamentos se referem as aplicações de uma movimentação financeira.

\section{CONSIDERAÇÕES FINAIS}

O propósito desse trabalho é servir como fonte de pesquisa aos interessados em conhecer o cenário dos empreendedores na busca por soluções aos seus desafios de gestão. A partir deste artigo é conveniente a discussão mais específica sobre a Inteligência de Negócios no âmbito de cada região em particular, ao mesmo tempo que este possa estimular o desenvolvimento de aplicativos mais didáticos para esse público em questão.

A principal percepção obtida na pesquisa foi de que, os aplicativos que já estão no mercado possuem um sistema bem estruturado de componentes como controle de custos, gerenciamento de receitas, além de um sistema de administração, permitindo aos usuários caminharem na organização de seus negócios, adequando-se ao mundo da Inteligência de Negócios. Entre os sistemas computacionais avaliados, notou-se que o PLANILHAS, do sistema Operacional Android, apresentou uma abordagem acessível ao ensino da Educação Financeira e à Inteligência de Negócios. PLANILHAS possui um diferencial, capaz de aproximar o público mais leigo à utilização do software, aliando a gestão do seu empreendimento com a busca por conhecimento técnico basilar.

Os desafios do mercado de trabalho se dão a partir da formação inicial como cidadão, os quais muitas vezes não conseguem ser superados, mesmo após a criação de um negócio próprio. $\mathrm{Na}$ tentativa de preencher essas lacunas, muitos recorrem aos aplicativos para celular.

A utilização de aplicativos como ferramenta de gestão financeira se mostrou muito adequada, uma vez que está presente no dia a dia de todos, estimulando e facilitando seu acesso, pois o usuário consegue adquirir informações de seu empreendimento à medida que alimenta o aplicativo com dados relevantes.

Espera-se que esse artigo sirva como exemplo e inspiração para o desenvolvimento de programas que facilitem a vida das pessoas, aprimorando o seu acesso à informação de qualidade mediante fontes fidedignas, mostrando que a Educação Financeira é um elemento essencial ao aprimoramento social. 


\section{RECIMA21 - REVISTA CIENTÍFICA MULTIDISCIPLINAR ISSN 2675-6218}

Como futuros trabalhos pode-se afirmar que as equações da Matemática Financeira apresentadas nesse artigo estão sendo escritas em Linguagem PYTHON de Computação. Mediante essa linguagem os modelos matemáticos poderão incorporar processos mais complexos, de forma a implementar funções multiobjectivos para análises da Engenharia Econômica, como por exemplo, os da Econometria.

\section{AGRADECIMENTOS}

Agradecemos à Direção do IFPA Campus Ananindeua pelo incentivo e apoio logístico e ao Grupo de Pesquisa Gradiente de Modelagem Matemática e Simulação Computacional - GM²SC, pelas contribuições científicas.

\section{REFERÊNCIAS}

CHENG, C.; ZHONG, H.; CAO, L. Facilitating speed of internationalization: The roles of business intelligence and organizational agility. Journal of Business Research, v. 110. p. 95-103. 2020.

CostA, D. C. L.; COSTA, H. A. de O.; NEVES, L. P. Métodos Matemáticos Aplicados nas Engenharias via Sistemas Computacionais. 2nd ed. Belém, PA: SINEPEM-IFPA, 2019.

COSTA, D. C. L.; COSTA, H. A. de O.; SILVA, H. C. M. da; SILVA, S. T. T. da. Matemática Computacional Aplicada à Ciência e Tecnologia. Belém, PA: SINEPEM-IFPA, 2021.

COSTA, D. C. L.; PEREIRA, M. O.; PINTO, C. A. L. Estruturas de Matemática Financeira Implementadas em PLANILHAS Eletrônicas. 5 Simpósio Internacional de Pesquisa em Educação

Anais...

SIPEMAT.

Disponível

em:

http://sipemat2018.sbempara.com.br/files/Anais SIPEMATParte2.pdf. 2018.

DANTAS, J. C. Contribuições da Implantação do Scrum como Metodologia Ágil para a Otimização da Gestão de Projetos nas Organizações. RECIMA21 - Revista Científica Multidisciplinar, v. 2, n. 7, e27541, 2021. ISSN 2675-6218. DOI: https://doi.org/10.47820/recima21.v2i7.541

DAU, G. Número de trabalhadores autônomos bateu recorde no segundo trimestre de 2021. Rede Jornal Contábil, 2021. Disponível em: https://www.jornalcontabil.com.br/numero-detrabalhadoresautonomos-bateurecorde-no-segundo-trimestre-de-2021/

DUBEY, R.; GUNASEKARAN, A.; CHILDE, S. J. Big data analytics capability in supply chain agility. Management Decision, v. 57, n. 8, p. 2092-2112, 2019. DOI: https://doi.org/10.1108/MD-01-2018$\underline{0119}$.

EL-ADAILEH, N. A.; FOSTER, S. Successful business intelligence implementation: a systematic literature review. Journal of Work-Applied Management, v. 11, n. 2, p.121-132, 2019. DOI: 10.1108/JWAM-09-2019-002.

IE. Por que $89 \%$ dos estudantes chegam ao final do Ensino Médio sem aprender o esperado em matemática? Ribeirão Preto, SP: Escola da inteligência educação socioemocional, 2021 Disponível em: https://escoladainteligencia.com.br/blog/por-que-89-dos-estudantes-chegam-ao-final-do-ensinoedio-sem-aprender-o-esperado-emmatematica/.

MACEDO, A. C.; OLIVEIRA, D. V. D.; ARAÚJO, K. T.; RIBEIRO, S. S.; DEUS, C. F. Gestão Financeira através do Aplicativo Mobills. Revista científica eletrônica de ciências aplicadas FAIT, 2015. 


\section{RECIMA21 - REVISTA CIENTÍFICA MULTIDISCIPLINAR ISSN 2675-6218}

MOTERLE, S.; WERNKE.; JUNGES, I. Conhecimento Sobre Gestão Financeira dos Dirigentes de Pequenas Empresas Do Sul De Santa Catarina. Revista de Administração, Contabilidade e Economia - RACE, v. 18, n. 1, 2019.

NASCIMENTO, R. S. B. do. A Modelagem Matemática como Proposta Pedagógica na Perspectiva do Ensino da Matemática em Ambientes Informatizados no Ensino Médio. RECIMA21 - Revista Científica Multidisciplinar, v. 2, n. 11, e211905, 2021. ISSN 2675-6218. DOI: https://doi.org/10.47820/recima21.v2i11.905.

OLIVEIRA, E. dos S.; LIMA, T. A. M.; SILVA, M. de O.; SILVA, P. O. da. O Uso dos Softwares na Educação Matemática. RECIMA21 - Revista Científica Multidisciplinar, v. 2, n. 5, e25320, 2021. ISSN 2675-6218. DOI: https://doi.org/10.47820/recima21.v2i5.320.

PEREIRA, A. S.; SHITSUKA, D. M.; PARREIRA, F. J.; SHITSUKA, R. Metodologia da pesquisa científica. Santa Maria, RS: Núcleo de Tecnologia Educacional - UFSM, 2018.

RASIWALA, F. S.; KOHLI, B. Artificial Intelligence in FinTech: Understanding Stakeholders Perception on Innovation, Disruption, and Transformation in Finance. International Journal of Business Intelligence Research, v. 12, p. 48-65, 2021. DOI: 10.4018/IJBIR.20210101.oa3.

SEBRAE. Micro e pequenas empresas geram 27\% do PIB do Brasil. [S. I.]: Serviço Brasileiro de Apoio às Micro e Pequenas Empresas (SEBRAE). Disponível em: https://www.sebrae.com.br/sites/PortalSebrae/ufs/mt/noticias/micro-e-pequenas-empresas-geram-27do-pib-do-brasil,ad0fc70646467410VgnVCM2000003c74010aRCRD.

SILVA NETO, G. O.; MACÊDO, E. A.; OLIVEIRA, C. G. Estimulando Gestão Financeira Pessoal com Aplicativo. Anais... Simpósios de Informática do IFNMG - Campus Januária, 2018.

SILVA, A. C. B. S.; COELHO, B. M. L. Aplicativos De Gestão Financeira: um estudo exploratório. Revista Pesquisa em Administração (Caruaru-PE), 2020.

VAIO, A. D.; PALLADINO, R.; HASSAN, R.; ESCOBAR, O. Artificial Intelligence and Business Models in the Sustainable Development Goals Perspective: A Systematic Literature Review. Journal of Business Research, v. 121, p. 283-314, 2020. 